\title{
ON FUNCTIONS THAT COMMUTE WITH FULL FUNCTIONS
}

\author{
JON H. FOLKMAN ${ }^{1}$
}

A continuous function $f$ mapping the unit interval $[0,1]=I$ onto itself is said to be full if $I$ can be partitioned into a finite number of subintervals $J_{i}$ such that $f$ maps each $J_{i}$ homeomorphically onto $I$. The number of subintervals $J_{i}$ will be called the number of branches of $f$. H. Cohen [2] showed that two full functions which commute (under composition) must have a common fixed point. In [1] Baxter and Joichi investigated the question of which continuous functions commute with full functions. The author conducted a similar investigation independently, and in this note we give some extensions of the results in [1]. Henceforth, it will be assumed that all functions considered are continuous.

Following Baxter and Joichi we define a hat function to be a piecewise linear full function whose derivative has constant absolute value. A full function $f$ will be called regular if there is a homeomorphism $\phi$ of $I$ onto $I$ such that $\hat{f}=\phi f \phi^{-1}$ is a hat function. Baxter and Joichi show that if $g$ commutes with a hat function $f$ with at least two branches, then either $g$ is a hat function or $g$ is constantly equal to a fixed point of $f$. From this it follows that if $g$ commutes with a regular full function $f$ having two or more branches then $g$ is either full and regular or it is constantly equal to a fixed point of $f$.

An example is given in [1] of a full function with two branches which commutes with a nonconstant, nonfull function. This shows that the above result does not hold for irregular full functions. However, the following generalization is valid.

TheOREM 1. Let $f$ be a full function with $n \geqq 2$ branches. There is a continuous monotone increasing function $\phi$ mapping $I$ onto $I$ and $a$ hat function $\bar{f}$ with $n$ branches such that $\phi f=\bar{f} \phi$. If $g$ commutes with $f$, there is a continuous function $\bar{g}$ such that $\phi g=\bar{g} \phi$ and $\bar{g}$ commutes with $\bar{f}$. Furthermore, $\phi$ is a homeomorphism if and only if $f$ is regular.

Received by the editors May 5, 1965.

1 This research is sponsored by the United States Air Force under Project RAND -Contract No. AF 49(638)-700 monitored by the Directorate of Development Plans, Deputy Chief of Staff, Research and Development, Hq USAF. Views or conclusions contained in this Memorandum should not be interpreted as representing the official opinion or policy of the United States Air Force. 
Combining the results of Baxter and Joichi with Cohen's theorem, we see that if $g$ commutes with a regular full function $f$, then $f$ and $g$ have a common fixed point. Using Theorem 1 we obtain

THEOREM 2. Let $f$ be a full function. If $g$ commutes with $f$, then $f$ and $g$ have a common fixed point.

Proof of Theorem 1. For each positive integer $k$, let

$$
0=t(k, 0)<t(k, 1)<\cdots<t\left(k, n^{k}\right)=1
$$

be the points where $f^{k}$, the $k$ th iterate of $f$, assumes the values 0 and 1. Define $\phi$ by

$$
\phi(x)=\sup \left\{\frac{i}{n^{k}} \mid k>0,0 \leqq i \leqq n^{k}, t(k, i) \leqq x\right\} .
$$

Clearly $\phi$ is monotone increasing, $\phi(0)=0$ and $\phi(1)=1$. Since $t(k, i)$ $=t(k+1, n i), \phi(t(k, i))=i / n^{k}$. Hence, $\phi(I)$ is dense in $I$. But $\phi$ is monotone so this implies that $\phi$ is continuous.

Let

$$
T^{k}=\left\{t(k, i) \mid 0 \leqq i \leqq n^{k}\right\}
$$

and let $T=\cup_{k} T^{k}$. Then $T$ is the set of points in $I$ which are mapped in to $\{0,1\}$ by some iterate of $f$. Now $f$ is onto and $f(\{0,1\}) \subset\{0,1\}$ so $f(T)=f^{-1}(T)=T$. Furthermore, if $0 \leqq x \leqq y \leqq 1$, then a necessary and sufficient condition for $\phi(x)$ to be equal to $\phi(y)$ is that the interval $[x, y]$ contain at most one point from the set $T$.

Define $\bar{f}$ by

$$
\ddot{f}(x)=\phi f\left(\phi^{-1}(x)\right) .
$$

To show that $\bar{f}$ is well defined we must show that $\phi(x)=\phi(y)$ implies that $\phi f(x)=\phi f(y)$. Suppose not. Then there are $x, y \in I$ such that at most one point of $T$ is between $x$ and $y$ and two points of $T$ are between $f(x)$ and $f(y)$. But these two points are the images under $f$ of a pair of points between $x$ and $y$. This is a contradiction since $f^{-1}(T)$ $=T$.

We clearly have $\bar{f} \phi=\phi f$. Let $Q$ be a closed subset of $I$. Then $\bar{f}^{-1}(Q)=\phi\left(f^{-1} \phi^{-1}(Q)\right)$. Now $f^{-1} \phi^{-1}(Q)$ is closed and hence compact by the continuity of $f$ and $\phi$. Therefore, $\phi\left(f^{-1} \phi^{-1}(Q)\right)$ is compact and hence closed so $\bar{f}$ is continuous.

To show that $\bar{f}$ is a hat function with $n$ branches, it is sufficient to show that, for each $k>0$ and each $i, 0 \leqq i<n, \bar{f}$ maps the points $i / n+j / n^{k+1}, 0 \leqq j \leqq n^{k}$, monotonically on to the points $l / n^{k}, 0 \leqq l \leqq n^{k}$.

This follows from 


$$
\begin{aligned}
& \bar{f}\left(\frac{i}{n}+\frac{j}{n^{k+1}}\right) \\
& =\bar{f} \phi\left(t\left(k+1, n^{k} i+j\right)\right)=\phi f\left(t\left(k+1, n^{k} i+j\right)\right) \\
& \quad=\left\{\begin{array}{l}
\phi(t(k, j))=\frac{j}{n^{k}} \text { if } f \text { is increasing on }[t(1, i), t(1, i+1)], \\
\phi\left(t\left(k, n^{k}-j\right)\right)=\frac{n^{k}-j}{n^{k}} \text { if } f \text { is decreasing on }[t(1, i), t(1, i+1)] .
\end{array}\right.
\end{aligned}
$$

Now suppose that $g$ commutes with $f$. By the above argument, to show the existence of a continuous $\bar{g}$ satisfying $\phi g=\bar{g} \phi$ it suffices to show that $\phi(x)=\phi(y)$ implies $\phi g(x)=\phi g(y)$.

Suppose not. Then for some $x, y \in I$ with $x<y, \phi(x)=\phi(y)$ and $\phi g(x) \neq \phi g(y)$. If there is a point $t \in T$ with $x<t<y$, it is the only element of $T$ in $[x, y]$. Now $\phi(x)=\phi(t)=\phi(y)$ but either $\phi g(x) \neq \phi g(t)$ or $\phi g(t) \neq \phi g(y)$. Replacing $[x, y]$ by either $[x, t]$ or $[t, y]$, we may assume that there is no element $t \in T$ with $x<t<y$. Hence $f^{k}$ is monotone on $[x, y]$ for every $k$.

Since $\phi g(x) \neq \phi g(y)$, for $k$ sufficiently large there are an arbitrarily large number of consecutive points from $T^{k}$ between $g(x)$ and $g(y)$. Hence, for any $r$ there is a $k$ and a monotone sequence $x_{1}, \cdots, x_{r}$ of points between $g(x)$ and $g(y)$ such that $f^{k}$ alternately assumes the values 0 and 1 on this sequence. The sequence $x_{1}, \cdots, x_{r}$ is the image under $g$ of a monotone sequence $y_{1}, \cdots, y_{r}$ of points in $[x, y]$. To see this, let $a, b \in I$. Now $g([a, b])$ is a closed interval containing $g(a)$ and $g(b)$. Hence, if $c$ is between $g(a)$ and $g(b)$, then $c$ is the image under $g$ of a point between $a$ and $b$. Since $x_{1}$ and $x_{r}$ are between $g(x)$ and $g(y)$, there are points $y_{1}$ and $y_{r}$ between $x$ and $y$ with $g\left(y_{1}\right)=x_{1}$ and $g\left(y_{r}\right)=x_{r}$. Now $x_{2}$ is between $x_{1}=g\left(y_{1}\right)$ and $x_{r}=g\left(y_{r}\right)$, so there is a point $y_{2}$ between $y_{1}$ and $y_{r}$ with $g\left(y_{2}\right)=x_{2}$. Continuing in this fashion, we construct a sequence of points $y_{1}, \cdots, y_{r}$ in $[x, y]$ with $g\left(y_{i}\right)=x_{i}$ and $y_{i}$ between $y_{i-1}$ and $y_{r}$ for $1<i<r$. This is the required monotone sequence.

The function $f^{k} g=g f^{k}$ alternately assumes the values 0 and 1 on the sequence $y_{1}, \cdots, y_{r}$. Since $f^{k}$ is monotone on $[x, y]$, the sequence $f^{k}\left(y_{1}\right), \cdots, f^{k}\left(y_{r}\right)$ is monotone and $g$ alternately assumes the values 0 and 1 on this sequence. But $r$ may be arbitrarily large, so this contradicts the continuity of $g$.

Since $\phi$ is onto, to show that $\bar{f} \bar{g}=\bar{g} \bar{f}$ it suffices to show that $\bar{f} \bar{g} \phi$ $=\bar{g} \bar{f} \phi$. This follows from the relations $\bar{f} \phi=\phi f, \bar{g} \phi=\phi g$ and $f g=g f$.

To see the final remark, observe that if $\phi$ is a homeomorphism then 
$\phi f \phi^{-1}=\bar{f}$ so $f$ is regular. Conversely, if $f$ is regular then the set $T$ is dense in $[0,1]$. This implies that $\phi$ is $1-1$ and hence a homeomorphism.

Before proceeding to the proof of Theorem 2, we need a

Lemma. Let $f$ and $g$ be continuous functions from $[a, b]$ to $[a, b]$ which commute. If $f$ is monotone then $f$ and $g$ have a common fixed point.

Proof. If $f$ is decreasing it has a unique fixed point $x_{0}$. Now $f g\left(x_{0}\right)$ $=g f\left(x_{0}\right)=g\left(x_{0}\right)$ so $g\left(x_{0}\right)=x_{0}$. If $f$ is increasing, let $x_{0}$ be a fixed point of $g$. The sequence $\left\{x_{n}\right\}$ defined by $x_{n}=f\left(x_{n-1}\right)$ for $n>0$ is monotone, so it has a limit which is a common fixed point of $f$ and $g$.

Proof of Theorem 2. If $f$ has only one branch, then $f$ is monotone and the conclusion follows from the lemma. If $f$ has more than one branch then the hypotheses of Theorem 1 are satisfied. By [1] and $[2], \bar{f}$ and $\bar{g}$ have a common fixed point $x_{0}$. Let $[a, b]=\phi^{-1}\left(x_{0}\right)$. Then $\phi f([a, b])=\bar{f} \phi([a, b])=\bar{f}\left(x_{0}\right)=x_{0}$, so $f([a, b]) \subset[a, b]$. Similarly, $g([a, b]) \subset[a, b]$.

Suppose $f$ is not monotone on $[a, b]$. Then there is a $t \in T^{1}$ with $a<t<b$. Now $f(t) \in[a, b]$ and $f(t) \in T$, but $[a, b]$ contains at most one point from $T$, so $f(t)=t$. However, $f(t)$ is either 0 or 1 and neither of these points are in the interior of $[a, b]$. Hence, $f$ is monotone on $[a, b]$. By the lemma, $f$ and $g$ have a common fixed point in $[a, b]$.

\section{REFERENCES}

1. Glen Baxter and J. T. Joichi, On functions that commute with full functions, Nieuw. Arch. Wisk. (3) 12 (1964), 12-18.

2. H. Cohen, On fixed points of commuting functions, Proc. Amer. Math. Soc. (2) 15 (1964), 293-296.

RAND CORPORATION 